\title{
Awareness and Collaboration Across Stakeholder Groups Important for eConsent Achieving Value-Driven Adoption
}

Therapeutic Innovation \& Regulatory Science 2019, Vol. 53(6) 724-735 (C) The Author(s) 2019 Article reuse guidelines: sagepub.com/journals-permissions DOI: I0.II77/2I684790|986|924 tirs.sagepub.com

\author{
Hilde Vanaken, Ir, MsC, PhD', and Shirley N. Masand, PhD²
}

\begin{abstract}
Background: While the shift to digital technologies is pervasive across multiple industries, the informed consent process for clinical trials remains largely paper-based. Given the inefficiencies in the traditional process and the increasing complexity of clinical studies, the current approach at times may raise challenges with respect to quality, compliance, participant understanding, and trial retention. Electronic informed consent (eConsent) is an enabling clinical technology to potentially address these issues by using multimedia components to create an interactive participant experience and improve data quality.

Methods: The TransCelerate eConsent Initiative conducted a multifaceted engagement approach to better understand the perceptions, experiences, and concerns of impacted stakeholders, including participants, sites, ethics committees, and health authorities.

Results: While all stakeholders were supportive of the use of multimedia components to deliver study information, several stakeholder-specific concerns were noted. Participant feedback, as collected through surveys $(n=3045)$ and an advisory board ( $n$ $=10$ ), suggests that some participants may have data privacy concerns and a diversity of preferences for multimedia presentation. Site interviews $(n=9)$ suggest concerns related to additional operational activities and potential for technology failure. Health authorities' feedback, through nonbinding meetings, was geographically varied; ethics committee feedback, through interviews, suggests concern over impact on operational process changes.

Conclusions: While the appetite for eConsent is increasing, involved stakeholders and industry must continue to raise awareness, communicate, and collaborate to develop appropriate technological capabilities, regulatory pathways, and operational processes to clear the path for mainstream use of eConsent.
\end{abstract}

\section{Keywords}

TransCelerate, eConsent, Electronic Informed Consent, Clinical Research, Digital, Patient-Centric

\section{Background}

While the shift to digital technologies is pervasive across multiple industries, the informed consent process for clinical trials has been historically paper based. Along with the increasing complexity of clinical studies, informed consent forms (ICFs) have become more complex, lengthy, and technical. ${ }^{1,2}$ As a result, the traditional paper-approach has been shown to raise challenges with respect to patient/participant (hereafter referred to as participant) understanding ${ }^{3,4}$ and trial recruitment, ${ }^{5}$ and may raise challenges with quality, compliance, and trial retention.

Electronic informed consent (eConsent) is an enabling clinical technology to potentially address these issues. Using multimedia components (eg, audio, video, pictures/diagrams), eConsent creates an interactive experience for participants aligned with their preferences, needs, and learning styles (eg, auditory, visual). ${ }^{6}$ Variations of this technology have been shown to improve participant understanding, ${ }^{7,8}$ and thereby empower participants to more easily make an informed decision. Importantly, the use of eConsent does not replace the site-participant relationship, and the site continues to own the consenting process. As with traditional paper-based consent, all stakeholders work together, with different roles and responsibilities toward the common goal of supporting participant understanding and protecting participant safety.

The use of eConsent allows access to higher quality and richer data than available with traditional paper-based

\footnotetext{
'Janssen Clinical Innovation, Janssen Research \& Development, Beerse, Belgium

${ }^{2}$ Accenture Life Sciences, Berwyn, PA, USA
}

Submitted 26-Dec-2018; accepted 06-Jun-2019

\section{Corresponding Author:}

Hilde Vanaken, Janssen Clinical Innovation, Janssen Research \& Development, Turnhoutseweg 30, 2340 Beerse, Belgium.

Email: hvanaken@its.jnj.com 


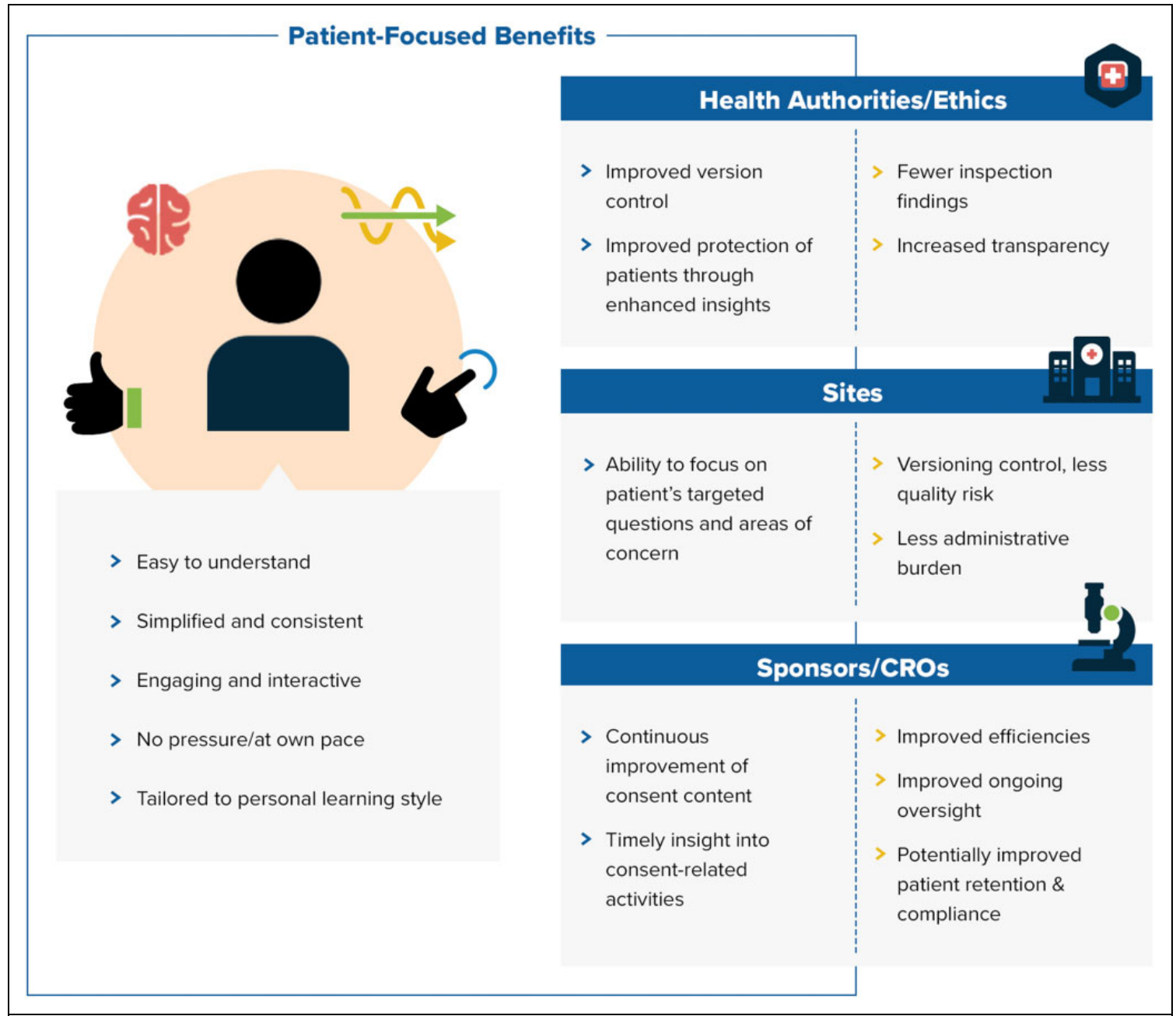

Figure I. Potential benefits of eConsent for key stakeholders. eConsent affords the opportunity to improve the consent experience and processes across clinical stakeholders, with the primary benefactor being the participant. The likelihood and degree of attaining these benefits is dependent on the content and context of eConsent implementation.

consent (Figure 1), which may improve the overall process for all stakeholders, including participants. ${ }^{9}$ For example, with eConsent, there is strict version control and an audit trail, which improves data quality and regulatory compliance and may reduce inspection findings (eg, wrong ICF version provided, missing/wrong dates). While the primary goal of using multimedia components is to empower participants to more easily make an informed decision, many of the multimedia components give richer insight into the participant consent experience (eg, flagging unfamiliar content and sections, use of glossary). ${ }^{9}$ Sites can use this information to tailor their conversation with the participant, and sponsors can have timely insight into eConsent activities and potentially use this aggregate experience data to improve the consent content. It is important to also note that the benefits seen from eConsent will be impacted by the content and context of the implementation.

Ultimately, eConsent may drive value in 2 ways for all involved stakeholders:
1. Empowers potential participants to more easily make informed decisions using interactive, multimedia components

2. Improves quality and efficiency of clinical trials through insight into the participant consent experience (eg, flagging unfamiliar content and sections, use of glossary), improved data quality, and a fully electronic system.

However, there are several barriers to broad implementation of eConsent oriented around the need for implementation considerations, awareness, and collaboration. First, there is a need to have an improved understanding of the processes required for global design and implementation of the technology. Also, there is a need to improve awareness and socialize that eConsent can potentially serve as a transformative method for delivering informed consent. Finally, to realize the efficiencies and benefits that eConsent can deliver to stakeholders, and most important to participants, changes in the ways of working will 
be required across multiple stakeholders, including participants, sites, health authorities, institutional review boards and ethics committees, participants, and sponsors; these changes are supported through effective collaboration.

The eConsent Initiative at TransCelerate Biopharma Inc. (TransCelerate) was initiated to address barriers to adopting eConsent by developing a cross-industry perspective on the implementation of eConsent. We used a multifaceted engagement approach to better understand the perceptions and concerns of impacted stakeholders. In this paper, we summarize the key insights from participants, sites, Health Authorities, and Ethics Committees, and provide approaches suggested by these stakeholders or that naturally follow from their insights.

The primary deliverables created through these activities include the eConsent Implementation Guidance, which describes the different eConsent multimedia components and implementation considerations, and an eConsent Global Landscape Assessment, which aggregates survey results about experience from pharmaceutical companies, and contract research organizations and vendors. For a full version of our library of assets, please visit the TransCelerate eConsent page at http://www.transceleratebiopharmainc.com/assets/ econsent/.

\section{Methods}

The TransCelerate eConsent Initiative worked over a 2-year period to compile and explore implementation experiences, lessons learned, and best practices regarding eConsent implementation and use. Throughout the development of the deliverables, the team actively engaged with external stakeholders. External stakeholders were selected based on convenience sampling and thus may not be representative of the views of the entire population, as statistical biases may be present. While it is noted that although most of the external engagement took place between 2015 and 2017, we updated our landscape assessment with data from 2018, which does not suggest any divergence or change from the original findings.

\section{Participant Feedback}

With the support of the Center of Information and Study on Clinical Research Participation (CISCRP), Clariness, CenterWatch, and TransCelerate, a 52-question online global participant survey was conducted from August to September 2016. Responses to 6 questions related directly to informed consent and eConsent are described. Questions were developed by TransCelerate eConsent team members. As this survey was executed digitally using the Internet, the survey responses represent a convenience sample, and findings may not be representative of the entire population.

Separately, TransCelerate commissioned CISCRP to develop an eConsent Participant Advisory Board with global representation. None of the Participant Advisory Board participants had prior experience with eConsent. CISCRP facilitated 3 semistructured focus group meetings with the
Participant Advisory Board: a virtual meeting in August 2016, a half-day face-to-face meeting in October 2016, and a virtual meeting in March 2017. During the half-day face-toface meeting, participants were given access to a sample eConsent tool. In alignment with focus group analysis methods, ${ }^{10}$ qualitative analysis methods were used to assess feedback. More specifically, analysis includes areas where individual opinions were expressed contradictory to group, minority opinions, and majority opinions.

\section{Site Feedback}

TransCelerate commissioned the Society for Clinical Research Sites (SCRS) to develop an eConsent Site Advisory Group. The Site Advocacy Group included 9 total representatives from 8 sites in the United States, Canada, United Kingdom, Australia, and Germany. None of the Site Advocacy Group participants had prior experience with eConsent. Site Advocacy Group sessions were grouped by geography across the following categories: North America, EU, and Asia Pacific. Four sessions were conducted in each geography virtually in May 2016, June 2016, September 2016, and March 2017. First and second sessions focused on understanding current experiences with informed consent and perceptions of eConsent. The third and fourth sessions focused on development and review of tools to support implementation of eConsent for sites (more information available at http://www.transceleratebiopharmainc.com/ assets/econsent/).

In addition to the eConsent Site Advocacy Group, the TransCelerate team conducted surveys and received written responses from 2 sites in Japan that had experience with eConsent pilots. Similar to the participant focus groups, Site Advocacy Group focus group feedback was analyzed using qualitative research methods. ${ }^{10}$

\section{Health Authority and Ethics Committee Feedback}

TransCelerate conducted informal, nonbinding face-to-face meetings with the following Health Authorities: European Medicines Agency (EMA of the European Union [EU], 2016), EMA Inspector's Working Group (IWG, 2017), Food and Drug Administration (FDA of the US, 2016), Federal Institute for Drugs and Medical Devices (BfArM of Germany, 2016), Pharmaceutical and Medical Devices Agency (PMDA of Japan, 2016), Taiwan Food and Drug Administration (TFDA of Taiwan, 2016), and Health Canada (HC, 2017).

TransCelerate also conducted virtual focus groups with Ethics Committees from the US and Germany, written surveys with Ethics Committees from Japan, and a face-to-face eConsent workshop in the UK including several Ethics Committees (jointly facilitated with the Health Research Authority).

It should be noted that the outcomes of these meetings do not reflect an official Health Authority or Ethics Committee stance. 
Table I. Demographic Characteristics of Participants Who Provided Feedback.

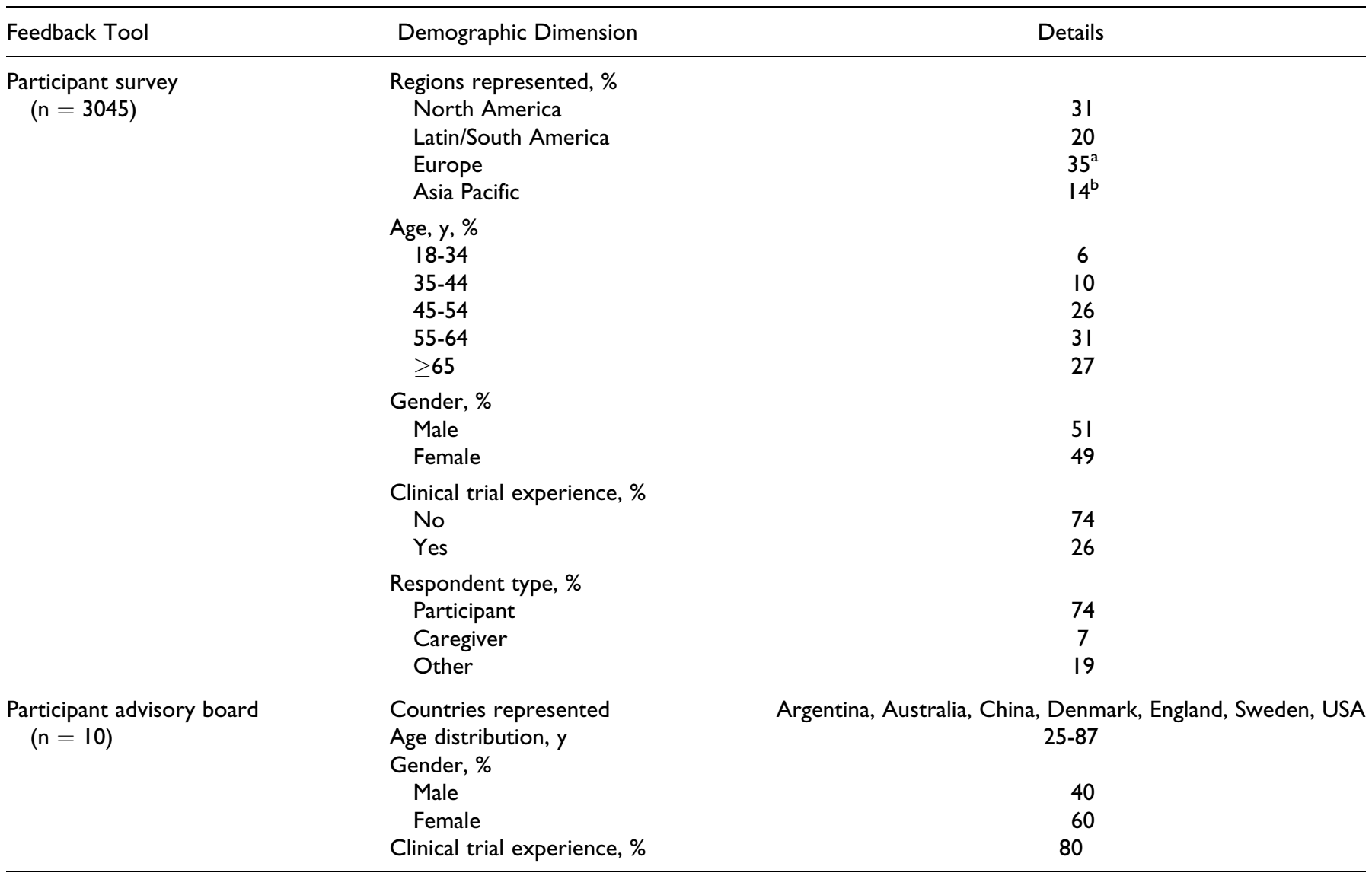

${ }^{\mathrm{a}} 89 \%$ from Germany.

${ }^{\mathrm{b}} 60 \%$ from Australia.

\section{Results}

\section{Participant Feedback}

Participant feedback came in the form of 3045 survey responses and 10 Participant Advisory Board participants (Table 1). Notably, over half (58\%) of survey respondents were $\geq 55$ years old.

Participant survey responses for the questions related to comfort with technology, and preferences for information presentation method in the informed consent process are shown in Figure 2. Most survey respondents were very comfortable $(59 \%)$ or comfortable $(33 \%)$ with technology. Furthermore, most respondents (73\%) felt an electronic system (ie, eConsent) would help them understand the clinical trial, with $53 \%$ preferring a combination of paper document and electronic system to review the consent content. In response to a related question, most respondents $(81 \%)$ wanted to take home a printed version of the signed consent document, with $41 \%$ preferring to also have the electronic version. Most respondents thought it would be very useful $(66 \%)$ or somewhat useful $(29 \%)$ to be able to highlight text or graphics they did not understand in an electronic informed consent system and then discuss these items with the study site staff. Notably, these responses were consistent across age, geography, and gender.

Participant survey responses related to signature and data privacy are shown in Figure 3. Overall, the majority of respondents were either very comfortable $(28 \%)$ or comfortable $(36 \%)$ with signing an electronic informed consent. However, there were notable regional differences: respondents in North America and Asia Pacific were more comfortable than those in the EU. Respondents were somewhat concerned $(36 \%)$ or very concerned $(21 \%)$ about the security of their data collected in an electronic database during the consent process. However, most (64\%) of these respondents would still participate in a clinical trial that collected their data in an electronic database during the consent process.

An analysis was also conducted that compared relevant survey responses from those who participated in a clinical trial and those who did not have trial experience. The summary of those findings in shared in Table 2. In summary, the largest differences were seen in the format of signed consent that the respondents wanted to take home. Survey respondents with trial experience preferred paper $(45.32 \%)$, whereas survey respondents without trial experience wanted paper and electronic (43.76\%). Interestingly, respondents with trial experience 


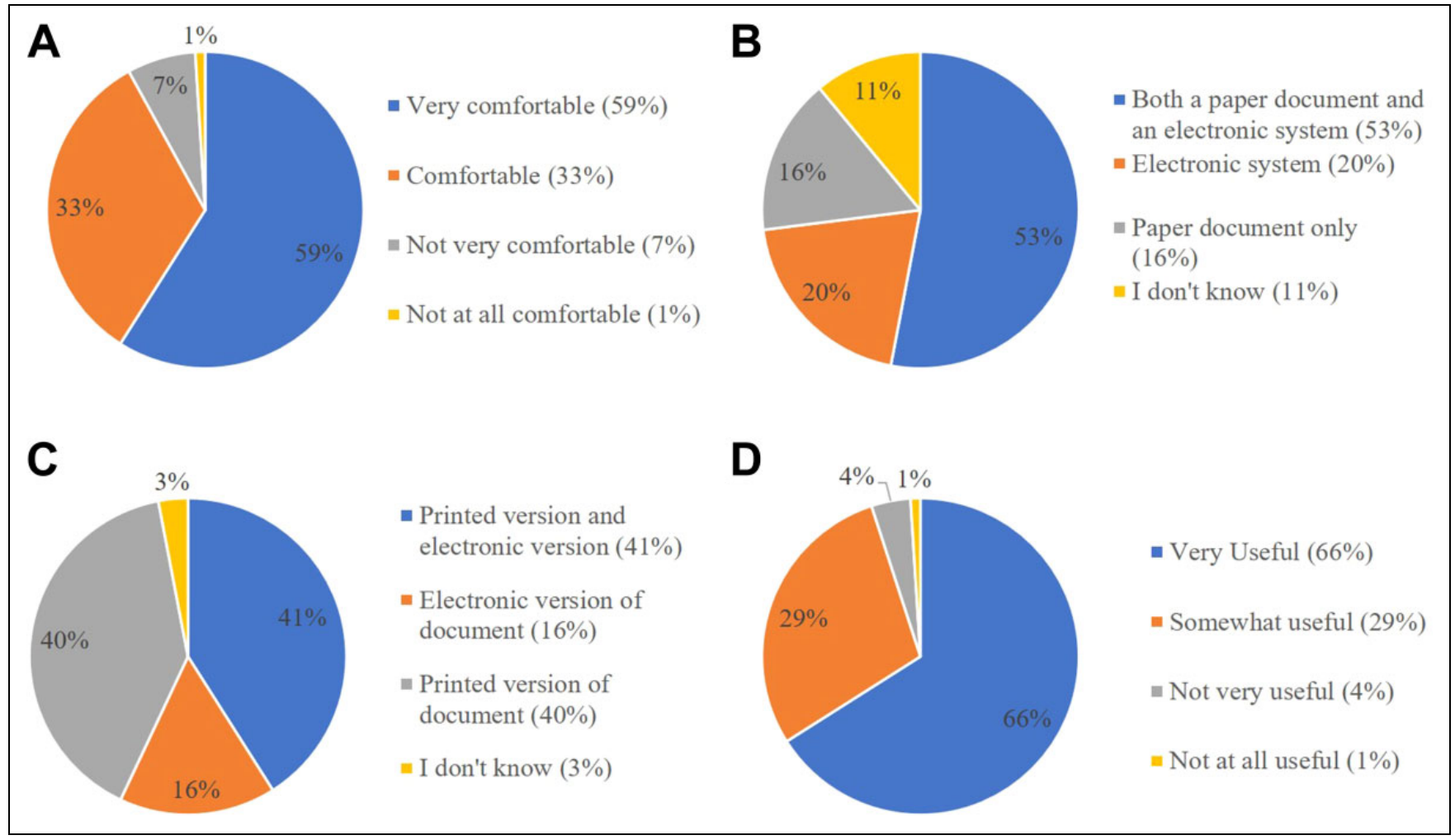

Figure 2. Global participant survey feedback on technology, informed consent delivery, and multimedia components. A global survey was completed by $n=3045$ participant respondents. The data in the figure includes respondent feedback as percentages on their (a) comfort with technology (eg, laptops, smartphones, tablets), (b) preference for whether the informed consent content is provided with paper or electronically, (c) the method for providing signed informed consent as a record back to the participant, and (d) usefulness of electronically highlighting text/graphics for follow-up discussion with study site staff or doctors.

showed significantly greater comfort with electronic signature ( $33.33 \%$ vs $28.21 \%$ ) and generally had less concerns with the security of their information if stored electronically.

In the Participant Advisory Board, participants were asked about their perceptions of eConsent before using or seeing a prototype. The majority of participants expressed that the multimedia components would help make the process more engaging and help the participants better understand the clinical trial process. For example, videos can be used to explain complex concepts. The majority of the participants also found the opportunity to ask study doctors and staff questions about the clinical trial valuable in improving their understanding and making an informed decision about participation. However, they were concerned that eConsent could impact the site/participant discussion - noting this concern was mitigated when the intent and process by which eConsent would be used was clarified. Some participants also expressed concern about the safety and security of their personal medical information because they were not sure who would have access to their data. Finally, some participants mentioned that access to technology and utility for participants with visual/auditory impairment could be potential barriers to eConsent.

The Participant Advisory Board later had an opportunity to use an eConsent prototype, and all participants found the prototype to be easy to navigate. The majority of the participants particularly liked the video/audio, summary boxes, glossary terms, and tiered organization (where essential elements are included in a concise main section and more detailed information in later subsections, as described in the Clinical Trials Transformation Initiative informed consent recommendations ${ }^{11}$ ) of the information. Participants had different preferences for specific characteristics of multimedia components. For example, some preferred cartoon images while others preferred photos. Additionally, they thought the visuals should reflect the diversity of the targeted participant population (eg, age, gender, ethnicity) for the study. Notably, the majority of the participants expressed that having simple, concise language, in addition to the multimedia components, is critical because a lengthy consent form can be difficult to understand. Some also thought eConsent may be particularly useful with vulnerable populations such as participants with low literacy levels. While the majority of participants were comfortable with information about their consent experience, like which words they looked up in the glossary or the amount of time spent on a specific section, being accessible to other stakeholders, a subset of participants suggested a preference to not see these data themselves as it may lead to biased behavior. After having seen and used the eConsent prototype, most 


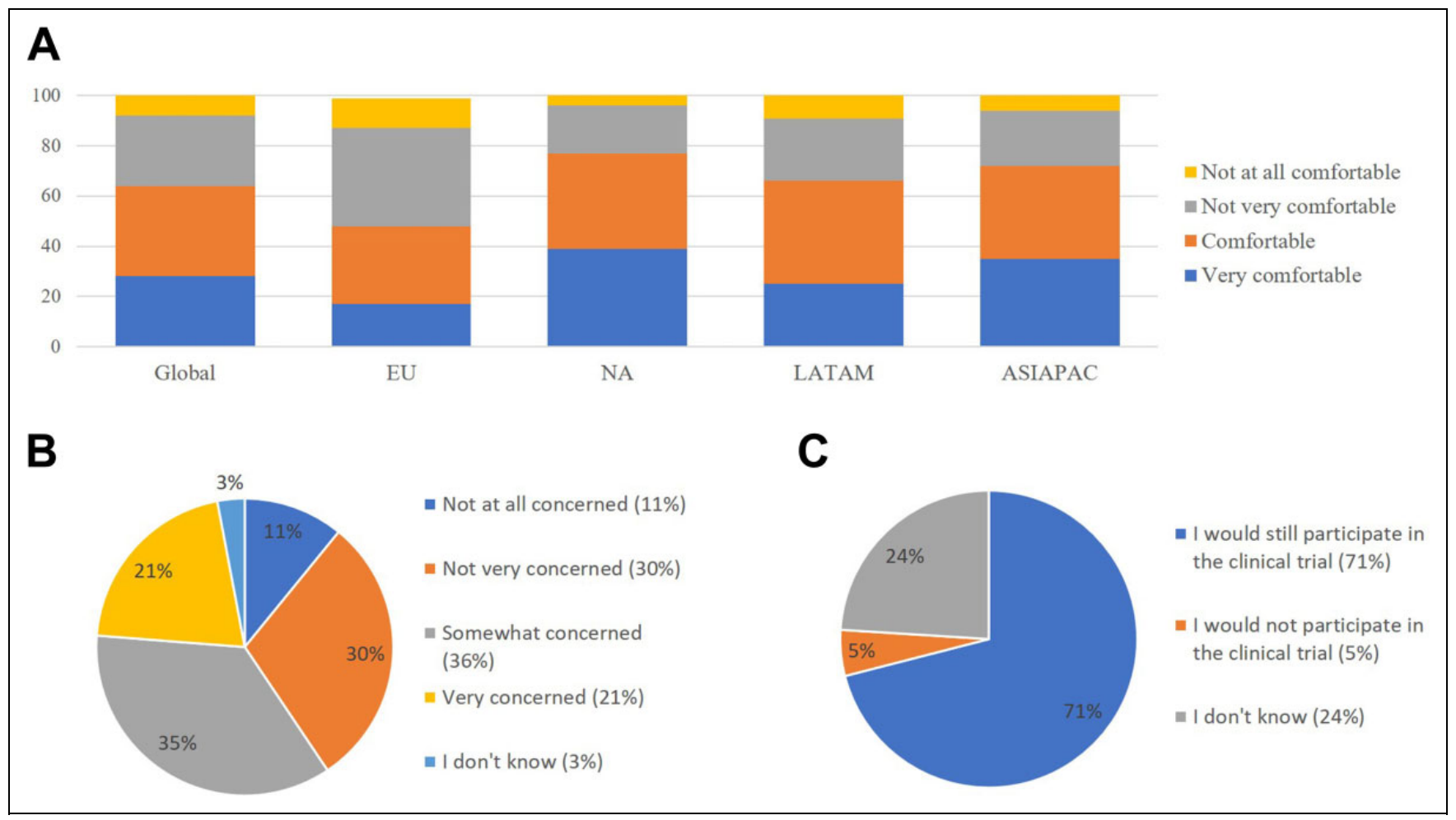

Figure 3. Global participant survey feedback on electronic signatures and data privacy considerations. A global survey was completed by $\mathrm{n}=$ 3045 participant respondents. The data in the figure includes respondent feedback as percentages.

Table 2. Comparison of Survey Results From Respondents With Trial Experience vs Without Trial Experience. ${ }^{a}$

\begin{tabular}{|c|c|c|c|}
\hline Survey Theme & Answer Choices & $\begin{array}{c}\% \text { With } \\
\text { Trial Experience } \\
\quad(n=80 \mathrm{I})\end{array}$ & $\begin{array}{c}\text { \% Without Trial } \\
\text { Experience } \\
(\mathrm{n}=2244)\end{array}$ \\
\hline \multirow[t]{4}{*}{ Method of delivery of consent information } & Paper document only* & 19.35 & 15.15 \\
\hline & Electronic system & 18.48 & 20.81 \\
\hline & $\begin{array}{l}\text { Both a paper document and an electronic } \\
\text { system }\end{array}$ & 53.56 & 52.45 \\
\hline & I don't know* & 8.61 & 11.99 \\
\hline \multirow{4}{*}{$\begin{array}{l}\text { Comfort in signing an informed consent } \\
\text { electronically }\end{array}$} & Very comfortable* & 33.33 & 26.38 \\
\hline & Comfortable & 33.46 & 37.25 \\
\hline & Not very comfortable & 25.72 & 28.16 \\
\hline & Not at all comfortable & 7.49 & 8.20 \\
\hline \multirow[t]{4}{*}{ Method for providing signed informed consent } & Paper* & 45.32 & 39.93 \\
\hline & Electronic* & 19.98 & 14.97 \\
\hline & Paper and electronic* & 32.33 & 43.76 \\
\hline & I don't know & 2.37 & 3.25 \\
\hline \multirow{5}{*}{$\begin{array}{l}\text { Concern with security of data if stored } \\
\text { electronically }\end{array}$} & Not at all concerned* & 25.34 & 19.03 \\
\hline & Not very concerned & 33.33 & 37.21 \\
\hline & Somewhat concerned & 30.59 & 29.46 \\
\hline & Very concerned & 8.99 & $\mid \mathrm{I} .4 \mathrm{I}$ \\
\hline & I don't know & 1.75 & 2.90 \\
\hline \multirow[t]{4}{*}{ Usefulness of multimedia components } & Very helpful & 64.42 & 66.71 \\
\hline & Somewhat helpful & 30.34 & 28.83 \\
\hline & Not very & 4.62 & 3.30 \\
\hline & Not at all & 0.62 & 1.02 \\
\hline
\end{tabular}

${ }^{\mathrm{a} C}$ Comparisons which were statistically significant by t-test are highlighted with an asterisk. 
participants had little or no concern about the security of their personal data but some remained concerned about who has access to their data.

For the future, most participants in the Participant Advisory Board wanted broad implementation of eConsent. As a part of this implementation, the Participant Advisory Board wanted the ability to reference the eConsent content, including multimedia components, throughout their participation in the trial. The majority also wanted a richer multimedia experience to further increase their understanding of the clinical trials (eg, more and improved multimedia components) and they desired personalized elements (eg, Dear Participant Name) within the eConsent form. Some also wanted to be able to record their questions electronically in the eConsent tool.

\section{Site Feedback}

Generally, the majority of site representatives supported the use of multimedia components to improve participant understanding. They felt the gathering of individual participant data as well as participant metadata though eConsent would allow more tailored site/participant engagement and enable continuous improvement of the eConsent content by the sponsor. However, one concern raised by most site representatives was wanting to ensure eConsent did not reduce site/participant interaction. Several site representatives, especially in the EU, had some concerns about the ability to comply with local regulations when collecting participant data such as electronic signatures.

Sites noted several benefits of eConsent technology: reduction in administrative burden (eg, eliminating the need for signing every page), reduction in paper trail, better version control, reduction in missing signature/date issues, improved data quality, and improved participant oversight (eg, need for reconsent). Site representatives were also supportive of participants having access to eConsent at home for review with family/ friends prior to signing because this capability could potentially reduce the number of site visits and participant burden. Their concerns centered mainly around technology issues (eg, loss of Internet and/or system failure) and the potential for increased workload (eg, extent of training, user access, and device management). They suggested that sponsors provide adequate training, documentation, and helpdesk support. The site representatives differed on the desired format (face-to-face, online, etc) of training.

Several of the sites indicated that they were already moving toward the use of more eClinical technologies (eg, ePRO). Sites expressed that they wanted user-friendly systems with early communication and collaboration between sites and sponsors. While some site representatives preferred a flexible approach, with eConsent having a paper backup, others were concerned that this hybrid approach could lead to a double consenting process.

For the future, site representatives envisioned broad implementation of eConsent with flexible device management (eg, single device for multiple eClinical apps or bring your own device $[\mathrm{BYOD}]$ ), and integration of eConsent with other
eClinical technologies such as randomization trial supply management (RTSM) and electronic data capture (EDC). They favored having the multimedia components of eConsent available to participants for home use, reconsenting by highlighting new content for participants, and having mutual recognition of eConsent training across sponsors to reduce the burden of having to take the same training multiple times for different sponsors.

\section{Health Authority Feedback}

All Health Authority representatives supported use of multimedia components to deliver study information. Some representatives specifically noted the benefit for vulnerable populations such as pediatric participants. Initial concerns about eConsent replacing site/participant discussions during the consent process were mitigated by further explanation that positioned eConsent as a tool to support, not replace, the site/ participant discussion. One Health Authority representative specifically suggested avoiding use of hyperlinks to websites that may change their content over time.

Representatives from some Health Authorities in the EU were concerned that eConsent may introduce additional site workload and that investigators/sites would have limited interest in the technology because they might need to use multiple systems within a single clinical trial. Also, representatives from multiple Health Authorities recommended that sponsors ensure participant comfort with eConsent technology.

Representatives from multiple Health Authorities had questions related to data access/archival, availability of data during inspections, source documentation, validation processes, and remote consenting. For example, they raised questions about access to documents after the eConsent process (eg, access to signed consent forms during an inspection). Representatives from several European Health Authorities recommended that sponsors discuss the potential use of eConsent in a trial with the Health Authorities before implementation to avoid unexpected issues arising at inspections.

The collection of participant data during the eConsent process was the topic of greatest concern to several Health Authority representatives at these face-to-face meetings. For example, some European Health Authority representatives were concerned about the use of electronic signatures and handling of signed eConsent forms. These concerns were about the site retaining control of the informed consent process and issues related to data privacy. They stated that the protection of participant data (name, signature) was the responsibility of the investigator and should not go beyond the site (ie, only coded or pseudo-anonymized data to be shared with the sponsor), unless controlled by the site. Any identifiable participant data sent outside the site should fall under the management of the investigator (not the sponsor). Further, they highlighted that sites would be responsible for vendor qualification and oversight. Given these concerns, remote monitoring of individual participants was not supported by representatives from several European Health Authorities. On the contrary, representatives 
of Health Authorities (including the US FDA) in other regions supported the use of electronic signatures, collection of individual participant data, and accessibility of data by remote monitoring. ${ }^{12}$ Representatives from these Health Authorities also noted the benefits (such as improved version control and fewer inspection findings) of technology.

Overall, all Health Authority representatives at these faceto-face meetings were in favor of broad implementation of eConsent in alignment with local regulations.

\section{Ethics Committee Feedback}

All Ethics Committee representatives supported the use of multimedia components to present study information but wanted to ensure use of such components was not coercive and that sponsors would bear the additional cost for using such technology. They had some concerns about the review process for the additional multimedia components, the impact on the review duration, and the format of the components for review. The stage of review was also a notable discussion: they wanted to review multimedia components in a draft stage that they could comment on but that included content reflective of the intended nature of the final interactive product. One Ethics Committee described receiving a video for comment when it was too late for any significant feedback to be incorporated. However, a storyboard provided earlier may not fully represent the intended content. A proposed solution was to send drafts for review with the opportunity to see the final component. Generally, Ethics Committees wanted collaboration with sponsors in developing the review process. Similar to Health Authority representatives, they suggested avoiding hyperlinks to websites with content that might change over time and had their initial concerns about eConsent replacing the site/participant consent discussion mitigated after learning more about eConsent as a tool to support this discussion.

Several Ethics Committee representatives had concerns about eConsent technology. Some noted that technologies are often not designed with the review process in mind (ie, difficult to make comments on electronic media) and pointed out the need for an updated, possibly technology-enabled review process. Some had the misperception that the eConsent device would be provided by the clinical site. They were concerned about the use of eConsent with participants not comfortable with technology, noting that these comments were addressed by highlighting that the paper consent would continue to be available as a backup.

Several Ethics Committee representatives specifically noted the benefits of collecting aggregate participant metadata to improve the consenting process, including improving the consent content. However, there were concerns of several Ethics Committee representatives over the collection of individual participant identifiable data, mirroring the concerns of some Health Authority representatives. Several Ethics Committee representatives, particularly those outside the US, were concerned about whether eConsent would comply with local regulations. Of note, Ethics Committee representatives outside of the US raised specific concern about the use of electronic signatures. Generally, representatives were also concerned about protection of participants' personal data, data access, and data archival.

Overall, Ethics Committee representatives, like Health Authority representatives, wanted broad implementation of eConsent in alignment with local regulations. They also wanted a technology-enabled electronic review/submission strategy for eConsent content.

\section{Discussion}

As with any new process or technology, successful eConsent implementation relies on the appropriate collaboration and communication with interested parties. To this end, these results provide information about the current state of experiences and perceptions with eConsent among participants, sites, Health Authorities, and Ethics Committees. The research in this paper suggests that these stakeholders have some commonalities, differences, and misconceptions in perceptions and expectations for eConsent.

Below, we present the key takeaways from TransCelerate's engagement with various stakeholder groups (participants, sites, Health Authorities, and Ethics Committees) across 5 topics:

- Implementation and use of technology

- Use of multimedia components to deliver study information

- Collection of participant data (eg, eSignature, metadata from multimedia components)

- Desired future state

- Implementation Considerations for Sponsors

\section{Implementation and Use of Technology}

Digital technologies have grown rapidly in recent years, and a significant portion of the world population uses these technologies in their day-to-day lives. eConsent is not a novel technology but rather an application of an existing technology in a novel setting.

Generally, stakeholders from all groups saw benefits to the use of technology to deliver study information, including reduction in administrative burden and the potential for reduction of participant burden by reducing the number of participant visits.

Several stakeholders expressed concern about the use of technology with elderly participants. Notably, our participant survey suggested that nearly all respondents $(>90 \%)$ were quite comfortable with technology, and $>50 \%$ of respondents were $\geq 55$ years old. Even with these results, there are certain populations who may not regularly interface with technologies. Therefore, it is important to ensure that the interface is userfriendly and that appropriate participant training is in place. In 
any case, the traditional paper informed consent is always available as backup.

While there were varied experiences with and perceptions of eClinical technologies across sites globally, the general concerns with the use of technology were consistent (eg, increased workload, technological issues, device management). At first, implementation of eConsent might introduce an additional system and activities, but this should be weighed against the benefits that it brings to the site and participant. In implementing eConsent, it is important to conduct an appropriate site feasibility assessment, including an assessment of technological capability. This assessment should establish the support the site will need for successful implementation. Throughout the process, sponsors should communicate early and often with sites so that sites have a full understanding of eConsent. As a part of this support, sponsors should ensure that there is appropriate training (in line with the site's preferred format), helpdesk support, and troubleshooting tips/manuals.

\section{Multimedia Components}

Stakeholders from all groups were unanimously supportive of the use of multimedia components to deliver study information. There was clear agreement that this would further empower participants to make informed decisions and allow for alignment with individual learning styles. However, within the Participant Advisory Board, we received varied feedback on specific preferences (eg, color, animation style, level of detail). Stakeholders from all groups also noted that specific consideration should be taken for vulnerable and pediatric populations. Designing a fit-for-trial eConsent requires understanding the participant population (eg, region, age, therapeutic area, culture) and learning preferences, and then choosing appropriate multimedia components. Sponsors may consider using focus groups to receive feedback on the design of the eConsent tool. Furthermore, in alignment with representatives from several Ethics Committees. and Health Authorities, it may be advisable to avoid the use of hyperlinks to websites with dynamic content, as the content of the destination webpage can change.

A common misconception from representative stakeholders from all groups was that eConsent could potentially reduce or eliminate the site/participant consent discussion. Integral to adoption of eConsent is the clear understanding that this technology is meant to be a supporting tool for sites in the consenting process. Therefore, clear communication of this concept early in the implementation process is highly desirable for all stakeholders. The reinforcement of this message should also occur in any training for the site and participant. In collaboration with the Site Advocacy Group, the TransCelerate eConsent Initiative has developed an "Introduction to eConsent" template, which provides a brief overview of what eConsent is, how it might work, and potential changes/considerations for sites; this template can be tailored to meet the particular needs or circumstances of specific sites or the particular sponsor use or trial.
While the use of multimedia components is a change for all stakeholders, the Ethics Committee representatives provided feedback that the electronic format of the consent will change the way they review it. Sponsors will need to work with Ethics Committees to develop review processes in line with their needs. This process will require an understanding of their technological capabilities and submission requirements as well as providing material that is still open for feedback yet appropriately depicts the participant experience.

Also, participants noted that while multimedia components are of high value, the content of eConsent should also be developed in alignment with health literacy principles. The TransCelerate eConsent Initiative has included some suggestions on how to incorporate health literacy principles into eConsent. More robust guidance on how to improve consent language has been developed by other organizations, including the Clinical Trials Transformation Initiative. ${ }^{11}$

\section{Collection of Participant Data}

Participants, sites, and Ethics Committees found value in the collection of individual and aggregated participant data/metadata to improve the overall consenting process. For example, the ability to understand where a participant spent a majority of their time during consent review might direct the site/participant discussion toward specific areas. Participants also expressed a desire to understand what data are being collected. Sponsors can best accommodate the participants' wishes by being transparent about what data are being collected, how the data are collected/protected, how long it will be stored, and what is being done with it.

For electronic collection and storage of participant data, the least acceptance across stakeholders was found in Europe. As a notable example, for eSignature, participant survey and Participant Advisory Board participants in Europe had the lowest level of acceptance (see Figure 3A; noting that $89 \%$ of the respondents were from Germany). Generally, there seemed to be regional lack of comfort with the collection of electronic data. Representatives of Ethics Committees in the EU also expressed concern about the ability of electronic data collection to comply with local regulations.

The largest barrier, however, is that some European Health Authorities may not be open to the collection of participant data (eg, participant name, participant signature) electronically, unless controlled and managed by the site. The reasons for this seemed to be multifold. As the site owns the consenting process, some Health Authority representatives felt that the site should oversee all consent-related activities, including vendor selection, and data collection/management.

These situations present significant challenges for eConsent. If participant data cannot be collected electronically and eConsent is limited to the use of multimedia components, the full benefit of eConsent will not be achieved. If enabling data collection requires the site to conduct vendor qualification and contracting, likely this will lead to a reduction in the use of 
Table 3. Feedback From Stakeholder Groups Toward Successful Implementation by Sponsors.

\begin{tabular}{|c|c|c|}
\hline $\begin{array}{l}\text { Stakeholder } \\
\text { Feedback }\end{array}$ & Feasibility & Design/Readiness \\
\hline Site & $\begin{array}{l}\text { - Conduct initial site communication/education to } \\
\text { discuss eConsent objectives and potential changes to } \\
\text { mitigate concerns } \\
\text { - Consider potential for integrations with other } \\
\text { eClinical technologies }\end{array}$ & $\begin{array}{l}\text { - Ensure interface is user-friendly } \\
\text { - Develop appropriate device management and } \\
\text { helpdesk approach } \\
\text { - Design appropriate operational (eg, how to use } \\
\text { metadata) and technical documentation and training, } \\
\text { in line with site preferences. } \\
\text { - Execute support activities including helpdesk and } \\
\text { troubleshooting manuals }\end{array}$ \\
\hline Participant & $\begin{array}{l}\text { - Tailor multimedia components to participant } \\
\text { population } \\
\text { - Collaborate with technology provider to assess if } \\
\text { enhanced multimedia components and expanded } \\
\text { capabilities could be included } \\
\text { - Consider methodologies to enable access to } \\
\text { eConsent content at home } \\
\text { - Consider integration with other participant } \\
\text { engagement platforms }\end{array}$ & $\begin{array}{l}\text { - Ensure interface is user-friendly and fit for } \\
\text { participants } \\
\text { - Conduct focus groups to receive feedback on } \\
\text { eConsent tools } \\
\text { - Ensure consent language is transparent about } \\
\text { participant data collection } \\
\text { - Develop training in line with participant population }\end{array}$ \\
\hline Health Authorities & $\begin{array}{l}\text { - Understand local regulations } \\
\text { - Consider preengagements with Health Authorities to } \\
\text { inform design }\end{array}$ & $\begin{array}{l}\text { - Communicate to understand if eConsent design is } \\
\text { compliant with local regulations } \\
\text { - Consider using workarounds if the collection of } \\
\text { participant data is not immediately achievable. }\end{array}$ \\
\hline Ethics committees & $\begin{array}{l}\text { - Consider manual and technology-enabled options for } \\
\text { review of submission materials }\end{array}$ & $\begin{array}{l}\text { - Collaborate with Ethics Committees to develop } \\
\text { review process in line with technological and } \\
\text { operational needs }\end{array}$ \\
\hline
\end{tabular}

eConsent in certain regions. Ultimately, the varied responses from the EU owing to local regulatory jurisdiction create barriers in developing a harmonized global implementation approach that allows participants and sites access to this technology.

As the regulatory uptake of eConsent is still dynamic, it is important to communicate and collaborate with local agencies and other official organizations (eg, privacy offices). When concerns are expressed, sponsors must take time to understand their root. These root concerns may be mitigated with information about validation, encryption, and other security measures. For example, highlighting that user access is restricted to specific and trained individuals might provide clarity into security processes in place. Furthermore, sponsors must be flexible to workarounds as this may be a multipart journey to realizing a fully electronic data collection. For example, in the near term, it may be necessary to consider an approach where multimedia components are delivered electronically, while signatures are still collected on paper in certain countries.

\section{Future State Considerations}

Representative stakeholders from all groups expressed the desire to see broader implementation of eConsent moving forward, with the caveat that this should always be in line with local regulations. Clearly, this is just the beginning of the journey for eConsent. To achieve a desirable future state for eConsent, collaboration with all involved stakeholders is critical (eg, participants, Ethics Committees, Health Authorities, sites, sponsors, vendors). While the following takeaways have varying degrees of technical feasibility, collaboration with technology providers may pave the way toward expanded capabilities.

While the Participant Advisory Board was supportive of eConsent, they expected to have a richer multimedia experience. In some ways, clinical technologies are behind the curve compared with other consumer-facing technologies. It will be critical in the design of participant-facing tools to incorporate good design principles and user experience testing to ensure sponsors are at pace with the digital world. There are numerous opportunities for eConsent to deliver advanced multimedia components, with simple (eg, ability to record participant questions) to more complex (eg, virtual reality of surgical procedures) features. Collaboration with eConsent technology providers will allow assessment of which advanced components are feasible for development.

The full benefits of electronic delivery of study information may not yet have been fully leveraged, including more efficient, tailored, and continuous communication. For example, the Participant Advisory Board and Site Advocacy Group expressed support for the ability to review eConsent, inclusive of the multimedia components, at home throughout the study. Sites supported providing participants with a tailored view of the reconsent that highlights the elements that have changed since the previous consent. These types of expanded capabilities are in alignment with eConsent's goal of keeping the participant informed and empowered. 
Particularly for sites and Ethics Committees, there is a standing concern regarding increased workload. Opportunities to introduce efficiencies are numerous and will enhance the likelihood of successful implementation. As a longer-term concept, flexible device management could potentially allow use of a single device for multiple eClinical apps or enable bring your own device approaches. Furthermore, eConsent also offers the opportunity for integrations with other eClinical technologies (eg, EDC) to increase process efficiencies and eliminate or reduce double-data entry. Finally, Ethics Committees expressed concerns about the review process for eConsent. Currently, the Ethics Committees are trying to fit a paper-based review into an electronic world. As an example, Ethics Committees use paper to provide comments on multimedia components. One method to add efficiencies to this process is to develop an authoring tool so that an Ethics Committee can directly include their comments into eConsent. As described in the section on multimedia components, Ethics Committees are seeking to review multimedia components early enough that comments can still be implemented, but that are reflective of the expected final content, and use of an authoring tool helps achieve this.

The informed consent process is often the first interaction the clinical trial participant has with the clinical trial process. It opens a world of opportunities by creating a digital connection with the clinical trial participant. Beginning with eConsent, sponsors could engage clinical trial participants through study reminders and personalized portals where information may be individually tailored. eConsent may support a rich study participant experience throughout the clinical trial and beyond.

\section{Implementation Considerations for Sponsors}

Taken together, impacted stakeholder considerations should be taken into account when implementing eConsent. In Table 3, the summary of feedback from stakeholder groups toward successful implementation is provided. These actions are organized into 2 phases, feasibility and design readiness, in alignment with the 4-phase approach outlined in TransCelerate's eConsent Implementation Guidance (http://www.transce leratebiopharmainc.com/assets/econsent/). The feasibility stage is when a sponsor is evaluating study considerations, defining desired multimedia components, reviewing operational considerations, reviewing data privacy/legal considerations, and defining stakeholder roles. The design/execution stage focuses on development and roll-out of eConsent, including regulatory submissions, ethics committee submissions, and site training.

\section{Conclusion}

The foundation of an effective informed consent process for clinical research studies is to provide study participants with the information that they need to make an informed decision on whether to participate in the study. eConsent empowers participants to make informed decisions using interactive multimedia components and enables improved quality and efficiencies across all stakeholders.

The industry is moving toward being more participant centric, designing trials around participants to improve their understanding and experience within the clinical trial. Consent is a big part of that, as it is one of the first key steps in a participant's decision to be in a trial. If the participants understand what is expected of them, then it stands to reason that they are more likely to be compliant with the study schedule and procedures. Furthermore, the shift to digital is pervasive across industries, including healthcare, and continues to be embedded in participant's day-to-day life. Participants expect convenient, easy-touse, professionally designed, and tailored digital solutions.

The research included in this paper has suggested that eConsent is an attractive and efficient option to deliver study information. Site representatives are generally supportive of using eConsent as a tool to support and enrich the site/participant discussion. Ethics Committees and Health Authorities generally understand that eConsent better informs participants about their potential trial participation. As the industry progresses in its adoption curve for eConsent, we all must keep in mind that the primary beneficiary of eConsent is the participant.

However, there are clear barriers and opportunities to be addressed to enable broad adoption of eConsent. The TransCelerate eConsent Initiative has developed tools to support adoption, including the Implementation Guidance and Landscape Assessment (http://www.transceleratebiopharmainc.com/assets/ econsent/). However, beyond the use of these tools, communication and collaboration with involved stakeholders is central to the success of eConsent. While TransCelerate continues to support eConsent through engagement in various forums and maintenance of publicly available deliverables, industry stakeholders will also have a role. Implementation insights (eg, lessons learned, potential improvements) should be broadly communicated to support objective-oriented improvements. Further, addressing topics like security and privacy will require multistakeholder dialogues, where holistic perspectives are considered to find mutually beneficial solutions. These dialogues should include patient representatives to ensure their perspectives are meaningfully understood and reflected. Moving forward, industry must continue to work together to develop appropriate technological capabilities, regulatory pathways, and operational processes to clear the path for mainstream use.

The future of participant-centric clinical trials inspires a more holistic, digitized, and integrated approach to participant engagement. eConsent is just the first step in this exciting journey.

\section{Acknowledgments}

The authors thank the broad membership of the TransCelerate eConsent Workstream for their support of this work. They also thank the patient, site, ethics committee, and Health Authority representatives who provided their inputs and perspectives.

\section{Declaration of Conflicting Interests}

No potential conflicts were declared. 


\section{Funding}

No financial support of the research, authorship, and/or publication of this article was declared.

\section{ORCID iD}

Shirley N. Masand, PhD (D) https://orcid.org/0000-0002-7345-1764

\section{References}

1. Hallian ZP, Forrest A, Uhlenbrauck G, Young S, McKinney R Jr. Barriers to change in the informed consent process: a systematic literature review. IRB Ethics Hum Res. 2016;38(3):1-10.

2. Paasche-Orlow MK, Taylor HA, Brancati FL. Readability standards for informed-consent forms as compared with actual readability. N Engl J Med. 2003;348(8):721-726.

3. Joffe S, Cook EF, Cleary PD, Clark JW, Weeks JC. Quality of informed consent in cancer clinical trials: a cross-sectional survey. Lancet. 2001;358(9295):1772-1777.

4. Falagas ME, Korbila IP, Giannopoulou KP, Kondilis BK, Peppas G. Informed consent: how much and what do patients understand? Am J Surg. 2009;198(3):420-435.

5. Ellis PM, Butow PN, Tattersall MHN, Dunn SM, Houssami N. Randomized clinical trials in oncology: understanding and attitudes predict willingness to participate. J Clin Oncol. 2001; 19(15):3554-3561.

6. Lentz J, Kennett M, Perlmutter J, Forrest A. Paving the way to a more effective informed consent process: recommendations from the clinical trials transformation initiative. Contemp Clin Trials. 2016;49:65-69.

7. Rowbotham MC, Astin J, Greene K, Cummings SR. Interactive informed consent: randomized comparison with paper consents. PLoS One. 2013;8(3).

8. Karunaratne AS, Korenman SG, Thomas SL, Myles PS, Komesaroff PA. Improving communication when seeking informed consent: a randomised controlled study of a computer-based method for providing information to prospective clinical trial participants. Med J Aust. 2010;192(7):388-392.

9. Vanaken H. eConsent study provides insights to shape industry adoption. Appl Clin Trials. 2016;25(8).

10. Kitzinger J. Qualitative research: introducing focus groups. BMJ. 1995;311(7000):299.

11. Clinical Trials Transformation Initiative: Informed Consent. https://www.ctti-clinicaltrials.org/files/ctti-informedconsentrecs.pdf.

12. FDA. Use of electronic informed consent in clinical investigations questions and answers guidance for industry. FDA. 2015;(March). 